\title{
Growth Pattern of Animals Is a Model for the Growth of Humans
}

\author{
V. L. Stass \\ Correspondence: V. L. Stass, Laboratory of animal genetics, institute of animal husbandry and veterinary science, \\ Latvia. e-mail: valbet.axon@apollo.lv
}

Received: January 4, 2021 Accepted: January 25, 2021 Online Published: January 26, 2021

doi:10.5539/jmr.v13n1p47

URL: https://doi.org/10.5539/jmr.v13n1p47

\begin{abstract}
The aim of this research was to translate a reading of a growth model of pigs to the modelling of growth of humans. The growth of individuals in the studied species is both a species-specific and phenotype-dependant process. The process is known as ontogenesis and has been considered as a dynamic system. The method used in the study was mathematical modelling. A hybrid model of animal's growth was applied to the experimental evidence to produce a reading of the growth dynamic. In the study, an earlier formulated analytical model of pig growth was developed to analyse a possible translation to the growth of humans. The study implies that in animals, the growth trajectory is phenotype-dependant, nonlinear and discontinuous. In some aspects such as saltatory growth and longevity as well as the discontinuous dynamic of the growth trajectory the translation of the results for the modelling the growth of humans were discussed.
\end{abstract}

Keywords: saltatory growth, ontogenetic trajectory, order parameter, dynamic system, hybrid model

\section{Introduction}

Models in mathematical biology aim to explain biological processes and then serve as a tool to promote new research in biology (Friedman, 2015). Modelling of growth of humans and animals has a long history, it goes back for about one hundred years. There are two different standpoints how to study growth of animals. One says that an observational rather than analytical approach is useful for improving our understanding of the phenomenon (Bünger et al, 2005). This is not the position taken in the study. Other position says that both analytical and experimental research combined may provide explanation of ontogenetic growth (Stass, 2019). In this study, the analytical model of animal's growth is the main aspect of the data analyses and the motive for the further study.

Each time we get into this log-jam of too much trouble, too many problems, it is because the methods that we are using are just the ones we have used before. The next scheme, the new discovery, is going to be made in a completely different way. There must be another way next time.

Richard P. Feynman, a distinguished physicist

\subsection{Models of Animal's Growth}

In research into growth, for over one hundred years long had dominated the growth data interpolation methods. Fitting growth curves to data was the mainstream in research into growth of animals and humans (Lee et al, 2020; Suki and Frey, 2017). Although the genetics of quantitative traits has been studied for over 100 years, very few of the polymorphisms that cause variation in these traits were known until recently (Goddard et al, 2016). Fitting curves to longitudinal phenotypic data is a common methodology that is used in animal and plant genetics to gain insights into individual growth patterns (Onogi et al, 2019). However, both in medicine and veterinary the species-specific growth models of individual organisms are of main importance. In the research, the growth interpolation functions deal with retrospective curve fitting and have neither predictive nor analytical potential. Growth of animals is a nonlinear process. Current evidence suggests that the normal process of growth is driven, at a physiological level, by growth itself and, at a molecular level, by a multiorgan genetic program. Current findings suggest that mammalian body growth is limited, at least in part, by a negative feedback loop (Lui and Baron, 2011).

\subsection{Genetic Determination of Ontogenetic Growth}

Growth is a quantitative trait. The growth and development are considered as a part of ontogenesis. In this study growth was modelled as the genetically determined trait. Since the beginning of genetics, there was a belief that quantitative and qualitative traits are determined in the same way, and the inheritable characteristics follow the same rules. Though, it turned out, that the phenotypes of quantitative traits are difficult to reveal. Still, it is thought that genetics of quantitative traits follow the same rules as the genetics of qualitative traits. Findings affirmed the extension of Mendelian concepts to 
complex traits, and there has been no looking back (Weiss, 2008). However, we know little about how the distribution of theoretically possible phenotypes originates through the process of ontogeny (Jaeger and Monk, 2014). Standard population genetic models simply assign phenotypic values directly to genotypes, without any causal explanation. While studying into the phenotypes of quantitative traits, there is a rule to follow: 'modelling of biological function has an essential role to play in unravelling genetic causation' (Noble, 2010).

While modelling a trait of an animal, we consider its phenotype. In animal, phenotype is any observable trait of interest in an organism. It follows that any morphological, developmental, biochemical, or physiological process or structure up to the subcellular level can be considered as phenotype (Mackay, 2009). It means that an organism's phenotype can be described by an effectively infinite number of traits, determined by a finite number of genes (Johnson and Barton, 2005). If infinite number of traits are determined by a finite number of genes then, according to the Dirichlet principle, there are traits, which are not genetically determined. That some traits in animal are not genetically determined is feasible. For example, there is no 'gene for' pacemaker rhythm (Noble, 2010). Some vital traits are not coded by DNA but are controlled by epigenetic mechanisms (van Baalen, 2013). In ontogeny, some biological structures and processes can emerge and disappear as dissipative systems (Goldbeter, 2018) that do not require genetic control. In this study we model animal's growth as a genetically determined trait. It has the consequence that the growth was modelled as a phenotype-dependant trait. In contrast, interpolation functions describe growth as an epigenetic trait.

\subsection{Growth Models of Humans}

Mathematical models for growth of humans are of the same kind as for animals. Fitting growth curves to data remain the standard method (Gliozzi et al, 2012). However, for clinical decisions in the context of precision medicine, we need individual growth prediction models rather than population based growth estimates. In clinical practice, individual growth trajectories would be needed to detect early signs of abnormal growth due to chronic disease, or to judge the efficiency of treatment strategies such as growth hormone therapy (Suki and Frey, 2017). There are many animals and from different species that serve as models for growth of humans. In the field of biomedicine, there is a tendency to use model animals of the comparable size, which are physiologically similar to humans. In this field, swine are anatomically and physiologically analogous to humans (Whyte and Prather, 2011). Both species are omnivorous, of comparable size, and similar digestive systems. Physiologically, pigs are comparable with humans in many respects; traits such as mature size, propensity to obesity, feeding patterns, dietary habits, digestive physiology are functionally similar in both species (Schook and Kuzmuk, 2011). Comprehensive studies of the skin, urinary, integumentary, and digestive systems demonstrate extensive similarities to humans. Swine may turn out to be the best alternative models (Watson et al, 2016).

\section{Methods}

In this study, an analytical model of pigs' growth (Stass, 2019) was developed. The model has been developed to analyse mainly qualitative aspects of growth, such as discontinuous dynamic and bifurcation of the growth trajectory. As well as the notion of phenotypes of the growth rate.

\subsection{The Model's Variables}

Let $\mathrm{M}$ denote an animal current live weight, measured in kilograms.

$M=\left\{M \in \mathbb{R}_{+} \mid 30 \leq M \leq 600\right\}$, an animal individual maximum weight $M_{x}=600 \mathrm{~kg}$.

$\mathrm{m}$ denotes an animal initial considered weight, measured in kilograms, $\mathrm{m} \leq \mathrm{M}, \mathrm{m}_{\mathrm{o}}=30 \mathrm{~kg}$.

Let $\mathrm{t}$ denote the chronological discrete current time, measured in days from animals' birth.

$\mathrm{t}=\{\mathrm{t} \in \mathbb{N} \mid 0 \leq \mathrm{t}<\infty\}, \Delta \mathrm{t}=1,2,3, \ldots, \mathrm{n} . \mathrm{n} \in \mathbb{N}$. $\mathrm{t}_{\mathrm{o}}$ denotes time related to $\mathrm{m}_{\mathrm{o}}, \mathrm{t}_{\mathrm{o}}=90$ days.

Let $K$ denote the invariant of growth, nondimensional. $K=\left\{K \in \mathbb{R}_{+} \mid 1 \leq K<11\right\}, K_{0}=1$.

Let $\mathrm{Z}$ denote the current feed conversion coefficient, nondimensional.

$Z=\left\{Z \in \mathbb{R}_{+} \mid Z_{0} \leq Z \leq \infty\right\},(Z=\infty) \rightarrow\left(M=M_{x}\right) v\left(M=M_{x x}\right)$, where $M_{x x}$ denotes species maximum weight.

Let $F$ denote consumed feed, measured in kilograms. $F=\left\{F \in \mathbb{R}_{+} \mid 0 \leq F<\infty\right\}$.

\subsection{The Model's Basic Functional Relations Between Variables}

The invariant of growth, parameter $\mathrm{K}$ has the following form

$$
K=\frac{M t}{m_{o}\left(2 t-t_{o}\right)} .
$$

An empirically found relation between the traits is given by system (2). 


$$
\left\{\begin{array}{l}
\frac{M}{m_{o}}=2 K-1+\frac{t-K t_{o}}{t} \\
\frac{t-K t_{o}}{t}=\frac{(Z-2 K)(K-1)}{Z K}
\end{array}\right.
$$

From (1) and (2) it follows

$$
\begin{gathered}
\frac{1}{m_{o}} \cdot \frac{\Delta M}{\Delta t}=\frac{1}{t} \cdot \frac{m_{o}[Z(2 K+1)-2 K]}{m_{o}[Z(2 K+1)-2 K]-M Z}, \\
\frac{1}{m_{o}} \cdot \frac{\Delta M}{\Delta K}=\frac{2 K+1}{K}-\frac{2}{Z} .
\end{gathered}
$$

The biological content of the above formulations is the following. Growth of an animal is considered a function of body weight $\mathrm{M}$, and the feed conversion coefficient $\mathrm{Z}$. The transformation of feed into an animal's weight is contingent on the current weight and the feed conversion coefficient. Parameter K denotes an invariant of the growth, and it makes the model species-specific.

\subsection{Methodological Aspects}

In the life sciences, modelling of biological systems by a single paradigm often is a difficult task due to intrinsic complexity of the systems. In many cases, the modelling paradigms are coupled in order to create hybrid methods (Smith and Yates, 2018). In the model, both continuum and discrete variables have been considered. As a result, standard continuum methods as well as a hybrid modelling approach were used. In both cases the growth of animals has been modelled as a dynamic system. The hybrid technique considers time as a discrete variable. In the model, the minimum discrete time $\Delta \mathrm{t}=1$ day corresponds to the duration of one cycle of the circadian rhythm, which oscillate with periods close to 24 hours (Goldbeter, 2018). During this time the physiological functions of the growth and development go through one cycle. In biological terms, during this period a consumed food has been transformed by an organism into human's or animal's weight.

\section{Results}

There are a few options to introduce a functional relation between the studied variables. In this section we consider both continuum and hybrid approach. In the model, continuum approach has grater generality but it is not easy to read. The hybrid technique invents a model with a clear biological logic yet with a limited capacity to be analysed.

\subsection{Continuum Approach for Modelling Some Aspects of Growth}

We can start with a well-known formula.

$$
\frac{M}{Z}=\int \frac{1}{Z} d M-\int \frac{M}{Z^{2}} d Z, Z>0
$$

We know that $\bar{Z}=\frac{F}{M}$, where $\bar{Z}$ denotes average feed conversion coefficient.

$$
\bar{Z}=\frac{M}{\int \frac{1}{Z} d M}, Z>0
$$

From (5) and (6) we have

$$
\begin{aligned}
& \frac{d \mathrm{~F}}{d M}=\frac{2 \mathrm{~F}}{M}-\frac{\mathrm{F}^{2}}{M^{2} Z}, \\
& \frac{d \bar{Z}}{d M}=\frac{\mathrm{F}}{M^{2}}-\frac{\mathrm{F}^{2}}{M^{3} Z} .
\end{aligned}
$$

Equations (7) and (8) give a general relation between current weight $\mathrm{M}$ and consumed feed $\mathrm{F}$. To provide transition from the general equations to the species-specific ones a species-specific parameter is needed. Modelling growth of pigs, as the species-specific variable has been used parameter K. Like in the general equations we use average feed conversion coefficient.

$$
\frac{\mathrm{F}}{M}=\frac{1}{K} \int Z d K
$$

From equation (9) it follows 


$$
Z=\frac{\mathrm{F}}{M}+\frac{K}{M} \cdot \frac{d \mathrm{~F}}{d K}-\frac{\mathrm{FK}}{M^{2}} \cdot \frac{d M}{d K}
$$

Equation (10) is species-specific, it shows how complicated the feed conversion coefficient $\mathrm{Z}$ is. Equations (7), (8) and (10) can serve as the first insight into some aspects of animal growth. A further modelling of growth is a target-dependant task. For example, in human infants growth is not continuous, it occurs in saltatory bursts separated by waiting periods of nearly no growth (Suki and Frey, 2017). Can we see periods of nearly no growth in a growing pig? Let us consider the invariant of growth, parameter K, equation (1). We have

$$
\frac{d K}{d t}=\frac{K}{M} \cdot \frac{d M}{d t}+\frac{\partial k}{\partial t}
$$

In complete form equation (11) is given by

$$
\frac{d K}{d t}=\frac{K}{M} \cdot \frac{d M}{d t}-\frac{K m_{O}}{M t} .
$$

In (12) consider $\mathrm{K}$ a constant, it means $\frac{d K}{d t}=0$. It follows

$$
\frac{K}{M}\left(\frac{d M}{d t}-\frac{m_{o}}{t}\right)=0,
$$

and we have

$$
\frac{M}{m_{o}}=1+\ln \left(\frac{t}{t_{o}}\right) .
$$

Equation (13) shows slow growth, nearly no growth under condition the growth invariant $\mathrm{K}$ is constant. This result is in full agreement with logic of growth process. One more option to consider slow growth is a stationary state in (11). From equation (11) under condition $\frac{\partial K}{\partial t}=0$, it follows

$$
\frac{M}{m_{o}}=K
$$

Equations (13) and (13.1) describe the growth, which is slower than average growth of an animal. Though, this growth is not necessarily abnormal. It can be the growth during a certain stage in development.

\subsection{A Hybrid Method for Modelling Growth of Animals}

From (1) and the system (2) one can derive the following equations.

$$
\begin{aligned}
\frac{1}{m_{o}} \cdot \frac{\Delta M}{\Delta t} & =\frac{1}{t} \cdot \frac{Z K(2 K+1)-2 K^{2}}{Z(K+1)-2 K}, Z_{o} \neq 1 . \\
\frac{\Delta K}{\Delta t} & =\frac{1}{t} \cdot \frac{Z K^{2}}{Z(K+1)-2 K}, Z_{o} \neq 1 .
\end{aligned}
$$

Both equations (14) and (15) in the point $\left.Z\right|_{K=1}=1$ are discontinuous. This point requires special attention. Biologically, $\mathrm{Z}=1$ means that all consumed food has been converted into animal's or human's weight, and this is hardly feasible.

Consider equation (14). If $\left.Z\right|_{K=1}=\frac{2}{3}$, then $\frac{\Delta M}{\Delta t}=0$. This means that animals do not grow if $Z=2 / 3$. When an animal reaches its individual maximum weight, under the model conditions it is $M_{x}=600 \mathrm{~kg}$, the growth stops. In this point $M=M_{x}, K=K_{x}, Z=Z_{x}$, and $t=t_{x}$. When growth stops, the feed conversion coefficient $Z_{x}$ grows into infinity $\left(Z_{x} \rightarrow \infty\right)$. We can find the growth rate under this condition. Consider the limit $\left(Z_{x} \rightarrow \infty\right)$ of equation (14).

$$
\lim _{Z_{x} \rightarrow \infty} \frac{\Delta M}{\Delta t}=\frac{m_{o}}{t_{x}} \cdot \frac{K_{x}\left(2 K_{x}+1\right)}{K_{x}+1} .
$$

In a more convenient form limit (16) is given below. 


$$
\frac{1}{m_{o}} \cdot \frac{\Delta M_{x}}{\Delta t_{x}}=\frac{1}{t_{x}} \cdot \frac{K_{x}\left(2 K_{x}+1\right)}{K_{x}+1}
$$

In the point $\left(M_{x}, K_{x}, Z_{x}\right)$ bifurcation takes place. Initially stable growth trajectory $m_{o} \rightarrow M \rightarrow M_{x}$, at the point $M=M_{x}$ loses its stability as $Z_{x}$ grows into infinity $\left(Z_{x} \rightarrow \infty\right)$. As a result, bifurcation shows up and the two new growth trajectories emerge (Stass, 2019). On one trajectory animals continue to grow till the species maximum weight $\mathbf{M}_{\mathrm{xx}}$ is obtained. On other trajectory animals continue to live till the obtainable life span is reached. In the bifurcation point the following change occurs $Z_{x} \rightarrow \infty \rightarrow Z_{x v}$, where $Z_{x v}$ denotes the feed conversion coefficient an instant after bifurcation, figure 2 .

\section{Discussion}

The biological sciences differ fundamentally from the physical sciences in their more balanced use of both deductive and inductive approaches to modelling. Both hierarchical and hybrid multiscale modelling method are expected to bring numerous benefits to biology (Coveney and Fowler, 2005). In this study, both continuum and hybrid modelling methods were used together. This combination of methods turned out to be efficient for modelling the growth of animals. The translation of the results for the modelling the growth of humans has been discussed.

\subsection{The Growth Trajectory of Animals}

The growth of animals is considered as a transformation of consumed feed into animal's weight. The efficiency of the feed transformation was modelled by the feed conversion coefficient $\mathrm{Z}$. In the model, the invariant of the growth $\mathrm{K}$, makes the growth process species-specific.

In this study, the growth of animals has been considered as a species-specific and phenotype-dependant process. In ontogeny, the increase of the weight of pigs is summarised in figure 1. The growth trajectory of some phenotypes is discontinuous. In this case, we analyse pitchfork bifurcation, which is a discrete, sudden, qualitative change in the trajectory (Roesch and Stumpf, 2019).

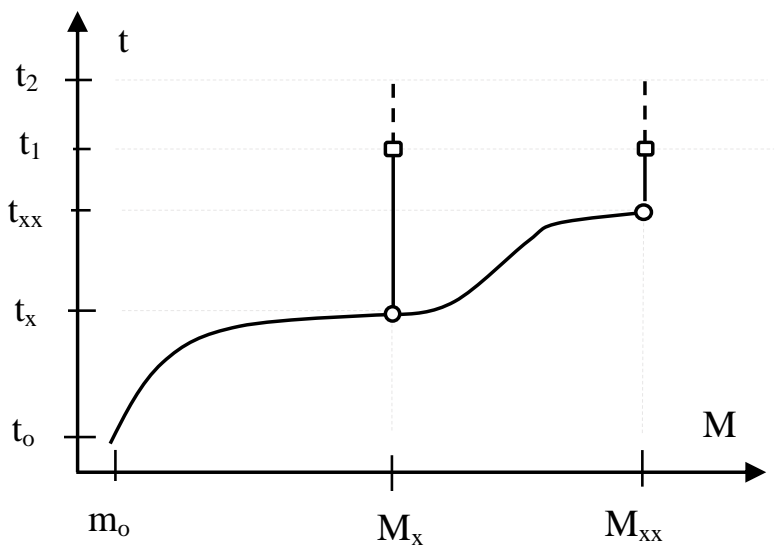

Figure 1. Growth trajectory bifurcation in animals' ontogeny. ${ }^{\circ}$ Bifurcation point. Bifurcation takes place in the point $\left(\mathrm{M}_{\mathrm{x}}\right.$, $\left.t_{x}\right)$ as the feed conversion coefficient $Z$ grows into infinity $\left(Z_{x} \rightarrow \infty\right)$. As a result, two new growth trajectories emerge. One growth trajectory is $M_{x} \rightarrow M_{x x}$. Other growth trajectory is $\left.\left.M_{x}\right|_{t x} \rightarrow M_{x}\right|_{t 1}$. Where $t_{1}$ denotes species obtainable life span 24,90 years, and $t_{2}$ denotes species maximum theoretical longevity 49,31 years

Bifurcation takes place in the point $\left(M_{x}, t_{x}\right)$, and $\left(M_{x x}, t_{x x}\right)$, figure 1(Stass, 2019). In these points the feed conversion coefficient $Z$ grows into infinity, $\left(Z_{x} \rightarrow \infty\right)$, and $\left(Z_{x x} \rightarrow \infty\right)$, respectively. We have to consider two more points, namely $\left(M_{x}\right.$, $\left.t_{1}\right)$, and $\left(M_{x x}, t_{1}\right)$, figure 1. In these points animals attain their individual obtainable life span $t_{1}=24,90$ years. It is thought that in the points bifurcation takes place as well. After bifurcation in the point $\left(\mathrm{M}_{\mathrm{x}}, \mathrm{t}_{\mathrm{x}}\right)$, two new growth trajectories emerge. The emerged trajectories are phenotype-dependant. It is thought that bifurcations in the points $\left(M_{x}, t_{1}\right)$, and $\left(M_{x x}\right.$, $\left.t_{1}\right)$, figure 1 are of the same kind as in the point $\left(M_{x}, t_{x}\right)$. Only certain phenotypes can take on the trajectory that leads to the species maximum theoretical life span $t_{2}$ from points $\left(M_{x}, t_{1}\right)$, and $\left(M_{x x}, t_{1}\right)$, figure 1 . Such selection of phenotypes takes place during the growth trajectory bifurcation. Second role of this bifurcation is regulate the growth. It is thought that this growth trajectory bifurcation initiates changes in animals' growth physiology to comply with the emerged trajectories.

After bifurcation, on one trajectory $\mathrm{M}_{\mathrm{x}} \rightarrow \mathrm{M}_{\mathrm{xx}}$ animals continue to grow till the species maximum weight $\mathrm{M}_{\mathrm{x}}$ is obtained, and later continue to live provided the weight remain constant. On other trajectory animals continue to live provided the weight $\mathrm{M}_{\mathrm{x}}$ remain constant. At bifurcation point, growth trajectory loses its stability as $\mathrm{Z}_{\mathrm{x}}$ grows into infinity and 
bifurcation shows up. The feed conversion coefficient $\mathrm{Z}$ is the order parameter, it causes the growth trajectory to bifurcate. The dynamic of the feed conversion coefficient $\mathrm{Z}$ is displayed in figure 2 .

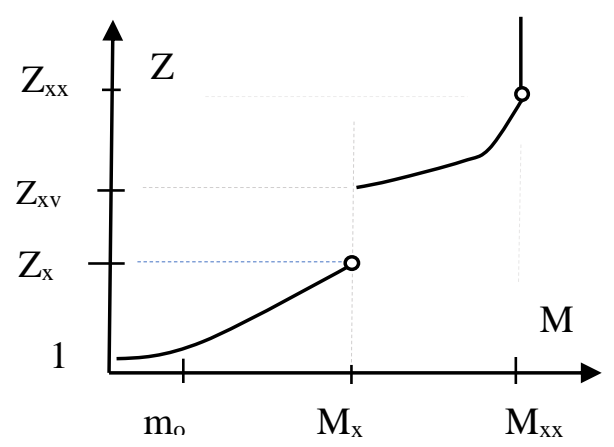

Figure 2. The feed conversion coefficient is both nonlinear and discontinuous function. $\circ$ Bifurcation point. Feed conversion coefficient $Z$ is the order parameter, it causes bifurcation to show up as $Z \rightarrow \infty$ at the point $\left(M_{x}, Z_{x}\right)$

In animals' ontogeny, there are two points where the growth function is discontinuous. In the point $\mathrm{Z}=\mathrm{Z}_{\mathrm{x}}$ the growth trajectory has bifurcation, and in the point $Z=1$ the function of growth rate is discontinuous. The two points in which the growth rate trajectory is discontinuous are shown in figure 3.

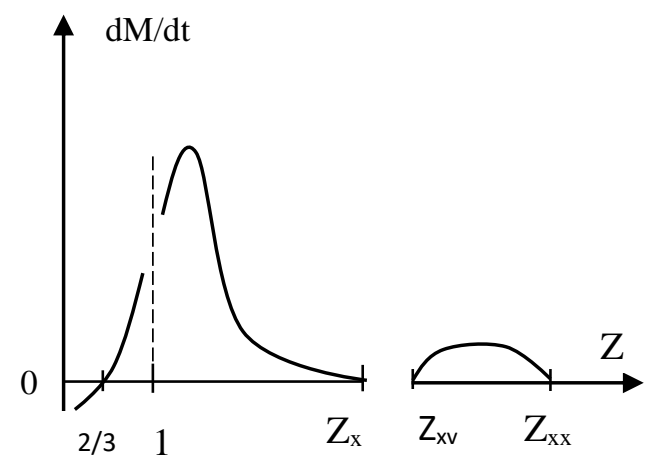

Figure 3 . The growth rate trajectory is a discontinuous function. The function is discontinuous in the points $\mathrm{Z}=1$, and $\mathrm{Z}=\mathrm{Z}_{\mathrm{x}}$ From equation (14) it follows that in the point $\left.Z\right|_{K=1}=1$ the function is discontinuous. There are some possibilities to consider. One possibility is that in the point $Z=1$ there is a local but not global maximum of the growth rate. It would be feasible during lactation, but hardly later. In ontogeny, $Z=1$ means that all food has been converted into weight, and this is hardly possible in the period after lactation. The value of the growth function in this point remains unclear. In the point $Z$ $=2 / 3$ animals do not grow. This result is unexpected and there is no straightforward explanation.

In the point $Z=Z_{x}$ bifurcation takes place. In an instant, the following change occurs $Z_{x} \rightarrow \infty \rightarrow Z_{x v}$. As a result, on one of two emerged growth trajectories animals continue to grow with $Z=Z_{x v}$ one instant after bifurcation, figure 3 . A normal form of this bifurcation is thought to be supercritical pitchfork bifurcation. It is a challenging task to describe this bifurcation in the normal form.

\subsubsection{The Notion of the Growth Phenotypes}

The notion of the growth phenotypes one can acquires from the analyses of the growth trajectory bifurcation. Consider figure 1 . Not all phenotypes can pass through the bifurcation point. Phenotypes that had not reached their maximum 
individual weight cannot enter this bifurcation. On the growth trajectory before the bifurcation point remain the phenotypes, which cannot reach their individual maximum weight. Through the bifurcation point can pass only some phenotypes. In animals' ontogeny, it is thought that bifurcation is a phenotype-dependant development. Animals take on one of the two emerged growth trajectories contingent on their phenotypes and not at random. This analysis suggests that one can distinct at least three sets with the growth phenotypes.

\subsection{A Translation to the Growth of Humans}

The growth of humans is to consider as a species-specific process. Humans grow slower than the most comparable size mammals. The reason for that slow growth is unclear. The growth of humans is not continuous. Observations of growth pattern in human infants provided evidence that growth is not continuous, it occurs in saltatory bursts separated by waiting periods of nearly no growth (Suky and Frey, 2017). The model of growth of animals has periods of nearly no growth. Such periods occur when the species-specific invariant of growth became a constant, equation (13), and (13.1). There is evidence that saltatory growth exists in infancy and even in school age although the exact distributions of saltatory intervals and growth amplitudes are still largely unknown (Suky and Frey, 2017). Analyses of a human-specific invariant of growth could help to clarify this aspect of man growth.

A reading of the model of animal's growth suggests that a number of features of growth in both species are similar. A theoretical relation between the traits is applicable to the growth modelling in both species, equations (5...10). In animals, a transition from a theoretical relation between variables to a species-specific one is obvious by introducing the species-specific invariant of growth, equation (10).

In both species, growth rate dynamic is similar in some aspects. During lactation, there can be a local growth maximum. After lactation a global phenotype-specific maximum during the rapid growth, and local maximum before puberty are feasible in the growth of humans as well.

Under the model conditions, growth trajectory bifurcation has two main functions. One is the shaping of the growth trajectory, and second function is the segregation of the growth phenotypes. In the course of growth, bifurcation is considered as a regular biological phenomenon, and not the species-specific event. In biological systems, nonlinear dynamic with bifurcations are known processes (Goldbeter, 2018; Roesch and Stumpf, 2019).

Phenotypes of the growth rate in animals is hardly unique phenomenon. One can expect a distribution of the growth phenotypes in humans as well. Understanding genetic variation is one of the keys to the development of personalized medicine. It is clear that genetic as well as phenotypic differences must be included in any patient-specific models (Coveney et al., 2013).

One of the possible applications of the model is insight into human longevity. In humans, both the theoretical longevity and obtainable life span remain unknown. Though, research into phenotype-dependant senescence is the method in which geriatricians have been interested for years. In animals, we can find both from the model. For example, obtainable life span in the pig is 24,90 years. This follows from the following analyses. Consider equation (4). There is a tiny, not definable border between $Z=Z_{x}$, and $Z_{x}=\infty$. The limit $(Z \rightarrow \infty)$ in (4) is given below.

$$
\lim _{Z \rightarrow \infty} \frac{\Delta M}{\Delta K}=\frac{m_{o} \cdot(2 K+1)}{K},\left.\left(K \rightarrow K_{1}\right)\right|_{Z \rightarrow \infty} .
$$

From (18) we get

$$
\frac{1}{m_{o}} \cdot \frac{\Delta M_{x}}{\Delta K_{1}}=\frac{2 K_{1}+1}{K_{1}} .
$$

It follows from (19) that $K_{1}=\left.K\right|_{(M=M x) \wedge(Z=\infty)}=10,04975$. Under condition $\left(K=K_{1}\right) \wedge\left(M=M_{x}\right)$ we have $t_{1}=24,90$ years, where $t_{1}$ denotes the species obtainable life span. In analytical form $t_{1}$ is given by (20).

$$
\frac{t_{1}}{t_{o}}=K_{1}^{2}
$$

It follows from the model that in the pig the maximum theoretical longevity $\mathrm{t}_{2}=49,31$ years (Stass, 2019).

This result suggests that growth and longevity are related traits. Moreover, these results support the opinion that the pathways controlling aging, also control growth, therefore suggesting that growth causes aging (Gems and Partridge, 2013). Ageing and lifespan are strongly affected by metabolism (van Beek et al., 2016). One can infer from the model that the feed conversion coefficient $\mathrm{Z}$ is the variable, which models metabolism. Under the model conditions variable $\mathrm{Z}$ delivers the coupling of functions between biochemical, and organism scales. The model opens up good chance for studying a relation between the growth, and aging by analytical methods. 


\section{Conclusions}

- Analytical modelling of growth of humans is to follow the same principles that have been used while modelling the growth of animals. A species-specific invariant of growth and the food conversion coefficient are to consider as the focal variables.

- In both species, the growth trajectory is thought discontinuous and nonlinear.

- The modelling of human's growth requires to know a relation between the growth invariant, the feed conversion coefficient, and the current weight.

○ In humans, a functional relation between the traits has to be found in physiological research or trials, and field observations.

\section{References}

Bünger, L., Lewis, R. M., Rothschild, M. F., Blasco, A., Renne, U., \& Simm, G. (2005). Relationships between quantitative and reproductive fitness traits in animals. Phil. Trans. R. Soc. B, 360, $1489-1502$. https://doi.org/10.1098/rstb.2005.1679

Coveney, P. V., \& Fowler, P. W. (2005). Modelling biological complexity: a physical scientist's perspective. J. R. Soc. Interface, 2, 267-280. https://doi.org/10.1098/rsif.2005.0045

Coveney, P. V., Diaz-Zuccarini, V., Graf, N., Hunter. P., Kohl, P., Tegner, J., \& Viceconti, M. (2013). Integrative approaches to computational biomedicine. Interface Focus 3:20130003. http://dx.doi.org/10.1098/rsfs.2013.0003

Friedman, A. (2015). Free boundary problems in biology. Phil. Trans. R. Soc. A, 373, 20140368. http://dx.doi.org/10.1098/rsta.2014.0368

Gems, D., \& Partridge, L. (2013). Genetics of Longevity in Model Organisms: Debates and Paradigm Shifts. Annu. Rev. Physiol., 75(6), 21-44. https://doi.org/10.1146/annurev-physiol-030212-183712

Goddard, M. E., Kemper, K. E., MacLeod, I. M., Chamberlain, A. J., \& Hayes, B. J. (2016). Genetics of complex traits: prediction of phenotype, identification of causal polymorphisms and genetic architecture. Proc. R. Soc. B, 283, 20160569. http://dx.doi.org/10.1098/rspb.2016.0569

Goldbeter, A. (2018). Dissipative structures in biological systems: bistability, oscillations, spatial patterns and waves. Phil. Trans. R. Soc. A, 376, 20170376. http://dx.doi.org/10.1098/rsta.2017.0376

Gliozzi, A. S., Guiot, C., Paolo, P., \& Iordache, D. (2012). A novel approach to the analysis of human growth. Theoretical Biology and Medical Modelling, 9, 17. https://doi.org/10.1186/1742-4682-9-17

Johnson, T., \& Barton, N. (2005). Theoretical models of selection and mutation on quantitative traits. Phil. Trans. R. Soc. B, 360, 1411-1425. //doi.org/10.1098/rstb.2005.1667

Jaeger, J., \& Monk, N. (2014). Bioattractors: dynamical systems theory and the evolution of regulatory processes. $J$ Physiol., 592(11), 2267-2281. https://doi.org/10.1113/jphysiol.2014.272385

Kuzmuk, K. N., \& Schook, L. B. (2011). Pigs as a model for biomedical sciences. The genetics of the pig, 2, $426-444$. https://doi.org/10.1079/9781845937560.0426

Lee, L., Atkinson, D., Hirst, A. G., \& Cornell, S. J. (2020). A new framework for growth curve fitting based on the von Bertalanffy Growth Function. Scientific Reports, 10, 7953. https://doi.org/10.1038/s41598-020-64839-y

Lui, J. C., \& Baron, J. (2011). Mechanisms Limiting Body Growth in Mammals. Endocrine Reviews, 32(3), $422-440$. https://doi.org/10.1210/er.2011-0001

Mackay, T. F. C. (2009). Q\&A: Genetic analysis of quantitative traits. Journal of Biology, 8, 23. https://doi.org/10.1186/jbiol133

Noble, D. (2010). Biophysics and systems biology. Phil. Trans. R. Soc. A 368. https://doi.org/10.1098/rsta.2009.0245

Onogi, A., Ogino, A., Sato, A., Kurogi, K., Yasumori, T., Togashi, K. (2019). Development of a structural growth curve model that considers the causal effect of initial phenotypes. Genet Sel Evol. 51, 19. https://doi.org/10.1186/s12711-019-0461-y

Roesch, E., \& Stumpf, M. P. H. (2019). Parameter inference in dynamical systems with co-dimension 1 bifurcations. $R$. Soc. open sci., 6, 190747. https://dx.doi.org/10.1098/rsos.190747

Suki, B., \& Frey, U. (2017). A time-varying biased random walk approach to human growth. Scientific Reports, 7, 7805.? 
Stass, V. L. (2019). A model of growth trajectory bifurcation in animals ontogeny, International Journal of Biology, 12(1), 20-29. https://doi.org/10.5539/ijb.v12n1p20

Smith, C. A., \& Yates, C. A. (2018). Spatially extended hybrid methods: a review. J. R. Soc. Interface, 15, 20170931. http://dx.doi.org/10.1098/rsif.2017.0931

van Baalen, M. (2013). Biological information: why we need a good measure and the challenges ahead. Interface Focus 3, 20130030. http://dx.doi.org/10.1098/rsfs.2013.0030

van Beek, J. H. G. M., Kirkwood, T. B. L., \& Bassingthwaighte, J. B. (2016). Understanding the physiology of the ageing individual: computational modelling of changes in metabolism and endurance. Interface Focus, 6, 20150079. https://dx.doi.org/10.1098/rsfs.2015.0079

Watson, A. L., Carlson, D. F., Largaespada, D. A., Hackett, P. B., \& Fahrenkrug, S. C. (2016). Engineered Swine Models of Cancer. Front. Genet., 7(78).

Whyte, J. J., \& Prather, R. S. (2011). Genetic Modifications of Pigs for Medicine and Agriculture. Mol. Reprod. Dev., 78, 879-891.

Weiss, K. M. (2008). Tilting at Quixotic Trait Loci (QTL): An Evolutionary Perspective on Genetic Causation. Genetics, 179, 1741-1756.?

\section{Copyrights}

Copyright for this article is retained by the author(s), with first publication rights granted to the journal.

This is an open-access article distributed under the terms and conditions of the Creative Commons Attribution license (http://creativecommons.org/licenses/by/4.0/). 